\title{
OPEN Chromosome-scale genome assembly for the duckweed Spirodela intermedia, integrating cytogenetic maps, PacBio and Oxford Nanopore libraries
}

\author{
Phuong T. N. Hoang $\mathbb{1}^{1,2,7}$, Anne Fiebig $\mathbb{1}^{1,7}$, Petr Novák $\mathbb{1}^{3}$, Jiři Macas $\mathbb{1}^{3}$, Hieu X. Cao $\mathbb{1}^{1,6}$, \\ Anton Stepanenko ${ }^{4}{ }^{4,5}$, Guimin Chen ${ }^{4,5}$, Nikolai Borisjuk ${ }^{4}{ }^{4,5}$, Uwe Scholz ${ }^{10}{ }^{1}$ \& \\ Ingo Schubert $\mathbb{1}^{1 \otimes}$
}

Duckweeds are small, free-floating, morphologically highly reduced organisms belonging to the monocot order Alismatales. They display the most rapid growth among flowering plants, vary 14-fold in genome size and comprise five genera. Spirodela is the phylogenetically oldest genus with only two mainly asexually propagating species: S. polyrhiza $(2 n=40 ; 160 \mathrm{Mbp} / 1 \mathrm{C})$ and S. intermedia $(2 n=36$; $160 \mathrm{Mbp} / 1 \mathrm{C})$. This study combined comparative cytogenetics and de novo genome assembly based on PacBio, Illumina and Oxford Nanopore (ON) reads to obtain the first genome reference for $S$. intermedia and to compare its genomic features with those of the sister species S. polyrhiza. Both species' genomes revealed little more than 20,000 putative protein-coding genes, very low rDNA copy numbers and a low amount of repetitive sequences, mainly Ty3/gypsy retroelements. The detection of a few new small chromosome rearrangements between both Spirodela species refined the karyotype and the chromosomal sequence assignment for S. intermedia.

Duckweeds are the smallest and fastest-growing flowering plants, and are considered as potential aquatic crops, serving for feed, food, fuel and waste water remediation ${ }^{1-13}$. They comprise 36 largely asexually propagating species within the 5 genera Spirodela, Landoltia, Lemna, Wolffiella and Wolffia ${ }^{14-16}$ With decreasing phylogenetic age duckweed frond sizes decrease from $1.5 \mathrm{~cm}$ to less than $1 \mathrm{~mm}$ in diameter accompanied by a successive reduction or loss of roots and a nearly 14 -fold genome size variation (from 160 to $2203 \mathrm{Mbp})^{17-20}$. These features make duckweeds an interesting subject for genome and karyotype evolution studies. So far, no correlation between genome size, chromosome number as well as ribosomal DNA loci was recorded from eleven species representative for five duckweed genera ${ }^{18}$.

The genus Spirodela harbors only two species of similar genome size (160 Mbp), S. polyrhiza and S. intermedia. Due to its basal ancestral phylogenetic position, its industrial potential and its small genome, the Greater Duckweed S. polyrhiza was chosen as the first duckweed for whole genome sequencing ${ }^{21}$. By integrating different approaches: cytogenetics, optical mapping (BioNano technique), Hi-C chromatin conformation study, 454, Illumina and Oxford Nanopore sequencing platforms, a high-confidence genome map for S. polyrhiza was established $^{22,24}$ that corrected the errors of previous genome maps ${ }^{21,23-25}$. This high-quality genome map provides a source for advanced genomic research regarding repetitive sequences and protein-coding genes, their chromosomal location and evolutionary history in other duckweeds. Moreover, whole-genome duplication (WGD) events and chromosomal rearrangement between duckweed species can potentially be uncovered using the $S$.

${ }^{1}$ Leibniz Institute of Plant Genetics and Crop Plant Research (IPK), 06466 Gatersleben, Stadt Seeland, Germany. ${ }^{2}$ Biology Faculty, Dalat University, District 8, Dalat City, Lamdong Province, Vietnam. ${ }^{3}$ Biology Centre, Czech Academy of Sciences, Institute of Plant Molecular Biology, České Budějovice 37005, Czech Republic. ${ }^{4}$ Jiangsu Key Laboratory for Eco-Agricultural Biotechnology Around Hongze Lake, School of Life Sciences, Huaiyin Normal University, Huai'an 223300, China. ${ }^{5}$ Jiangsu Collaborative Innovation Centre of Regional Modern Agriculture and Environmental Protection, Huaiyin Normal University, Huai'an 223300, China. ${ }^{6}$ Present address: Institute of Biology, Martin-Luther-University Halle-Wittenberg, 06120 Halle, Germany. ${ }^{7}$ These authors contributed equally: Phuong T. N. Hoang and Anne Fiebig. ${ }^{\square}$ email: schubert@ipk-gatersleben.de 
polyrhiza genome as a reference. Between the chromosomes of seven cytogenetically investigated S. polyrhiza clones so far no BAC-sized structural rearrangements were found ${ }^{24}$. In addition, population genomics studies suggested a considerably low genetic diversity between world-wide distributed S. polyrhiza clones ${ }^{26,27}$.

Sequence assignment to distinct chromosomes based on cross-hybridization of genomic sequences between related species represents a novel cytogenomic approach. Such an approach is particularly important for vegetatively propagating species, for which obtaining a genetic map to validate sequence assembly is difficult. However, in such cases at least one reference genome for validating bioinformatic assembly efforts is required, and a considerable number of cytogenetic anchor points should provide a reliable support for sequence data integration as previously exemplified for Amborella trichopoda ${ }^{28}$ and for S. polyrhiza ${ }^{23}$. These prerequisites are given for $S$. intermedia. In our previous study, chromosome homeology and rearrangements between S. polyrhiza $(2 \mathrm{n}=40)$ and $S$. intermedia $(2 \mathrm{n}=36)$ were investigated by cross-FISH with 93 anchor BACs of S. polyrhiza ${ }^{29}$. Thus, a highconfidence genome map of $S$. polyrhiza as a reference, and a cytogenetic map of $S$. intermedia are available to support genomic sequence assembly from reads generated by next-generation sequencing (NGS) platforms.

In this study, the cytogenetic maps of two S. intermedia clones (8410 and 7747) served as a frame for whole genome assembly of the vegetatively propagating $S$. intermedia. The same chromosomal rearrangements distinguishing clone 8410 from S. polyrhiza were also found for clone 7747 applying 93 anchored BACs. By integrating the cytogenetic maps and genome assembly from PacBio reads for clone 7747 and Illumina/ON reads for clone 8410 , we generated a robust, chromosome-scaled genome map, apparently identical for both $S$. intermedia clones and revealed additionally further small evolutionary rearrangements between the two Spirodela species.

\section{Results}

The cytogenetic map for S. intermedia clone 7747. Previously we established a cytogenetic map for $S$. intermedia clone 8410 using 93 BACs anchored in the $S$. polyrhiza genome ${ }^{29}$. Now we hybridized diagnostic BACs to the chromosomes of the $S$. intermedia clone 7747 in order to test whether chromosomal differences occurred between the karyotypes of these two $S$. intermedia clones, because different chromosome numbers $(2 n=36$ versus $2 n=20)$ have been reported for $S$. intermedia clone $7747^{30,31}$. Our current chromosome counting found no chromosome number difference between clones 7747 and 8410 . For both clones $2 n=36$ were counted ${ }^{18}$ (Fig. S1). In order to test whether structural chromosomal rearrangements occurred between these S. intermedia clones, we applied cross-FISH with suitable combinations of 93 S. polyrhiza BACs, as described in Hoang \& Schubert, $2017^{29}$, on mitotic spreads of $S$. intermedia clone 7747 . We used the same chromosome designation as in Hoang \& Schubert, 201729: ChrSp for chromosomes of S. polyrhiza and ChrSi for chromosomes of $\underline{\text { S. interme- }}$ dia. No chromosomal rearrangements between these two $S$. intermedia clones were detected. Six linkages in $S$. intermedia that differed from the S. polyrhiza karyotype [ChrSp03-ChrSp06-ChrSp14]; [ChrSp05-ChrSp06]; [ChrSp06-ChrSp07-ChrSp14]; [ChrSp03-ChrSp17], [ChrSp10-ChrSp16] and [ChrSp08-ChrSp18] previously reported for clone $8410^{29}$, were found also for clone 7747 (Fig. 1). The similarity between the cytogenetic maps of S. intermedia clones 7747 and 8410 enabled merging of genome assembly from PacBio reads for clone 7747 and of Illumina and ON reads for clone 8410 , and yielded an apparently identical genome map for both $S$. intermedia clones.

Genome assembly for S. intermedia clone 7747 based on the library of PacBio sequence reads. Two rounds of PacBio-sequencing of a $20 \mathrm{~kb}$ library of genomic DNA of S. intermedia clone 7747 resulted in $149 \mathrm{Gbp}$ of raw read data. After an initial filtering for potential bacterial contamination, reads of at least 500 nucleotides were assembled using the Canu pipeline v. $1.5^{32}$.

A total of 1,305,064 reads were assembled into 1172 sequence contigs of 147,613,042 nucleotides, corresponding $91.7 \%$ of the estimated genome size. All contigs of this draft assembly are covered in median 37.5 -fold by raw reads. In a first round of scaffolding, the two genomes of the sister species S. polyrhiza (from clones 9505 and $7498)^{23,25}$ were used as references to order contigs as described ${ }^{33}$. The resulting scaffolds (N50=1,7 Mbp) were super-scaffolded by SSPACE-Longread v.1-1 $1^{34}$ and assigned to the 18 chromosomes of S. intermedia, using 93 S. polyrhiza BACs as landmarks which were cross-hybridized to the S. intermedia chromosomes of clone $8410^{29}$. In addition to confirmation of the same linkage relationship in clone 7747 (Fig. 1), new cytogenetic probes using BACs from the genomic regions of interest were designed for FISH experiments to approve localization of the contigs within the pseudomolecules, to resolve mis-assemblies and/or to confirm new linkages (see below). Furthermore, contiguity of the assembly was confirmed by corrected ON reads of S. intermedia clone 8410 using minimap2 $2.16^{35}$ (see below).

After reiterative rounds of manual curation and validation by FISH, in the final genome assembly, 18 scaffolds ( $\mathrm{N} 50=8.3 \mathrm{Mbp}$ ) of in total $131.4 \mathrm{Mbp}(82.2 \%$ of the estimated genome size) could be assigned to the 18 chromosomes (Table 1). Six of them show telomeric sequences at both ends and seven at least on one end (see Fig. 2). Most of the shorter and/or repetitive sequences (16.2 Mbp, corresponding $10.1 \%$ of the estimated genome size) $(\mathrm{N} 50=27.1 \mathrm{Kbp})$ could not yet be assigned and were considered as additional pseudomolecule "SiUn".

Genome assembly for S. intermedia clone 8410 based on Illumina and Oxford Nanopore reads. The ON/Illumina-derived scaffolds for clone 8410 were created using the MaSuRCA assembler and filtered using minimap2 to remove duplicated sequences derived from heterozygous regions. To form pseudomolecules, all 70 remaining scaffolds were corrected and ordered by manual curation using the assembly of clone 7747 as a reference. Finally, all 18 pseudomolecules ended at both sites with telomeric sequences. Merging of both assemblies revealed further small rearrangements between the karyotypes of S. polyrhiza and S. intermedia (see below and Figs. 2 and 3). The quality of both S. intermedia genome assemblies was assessed by the 

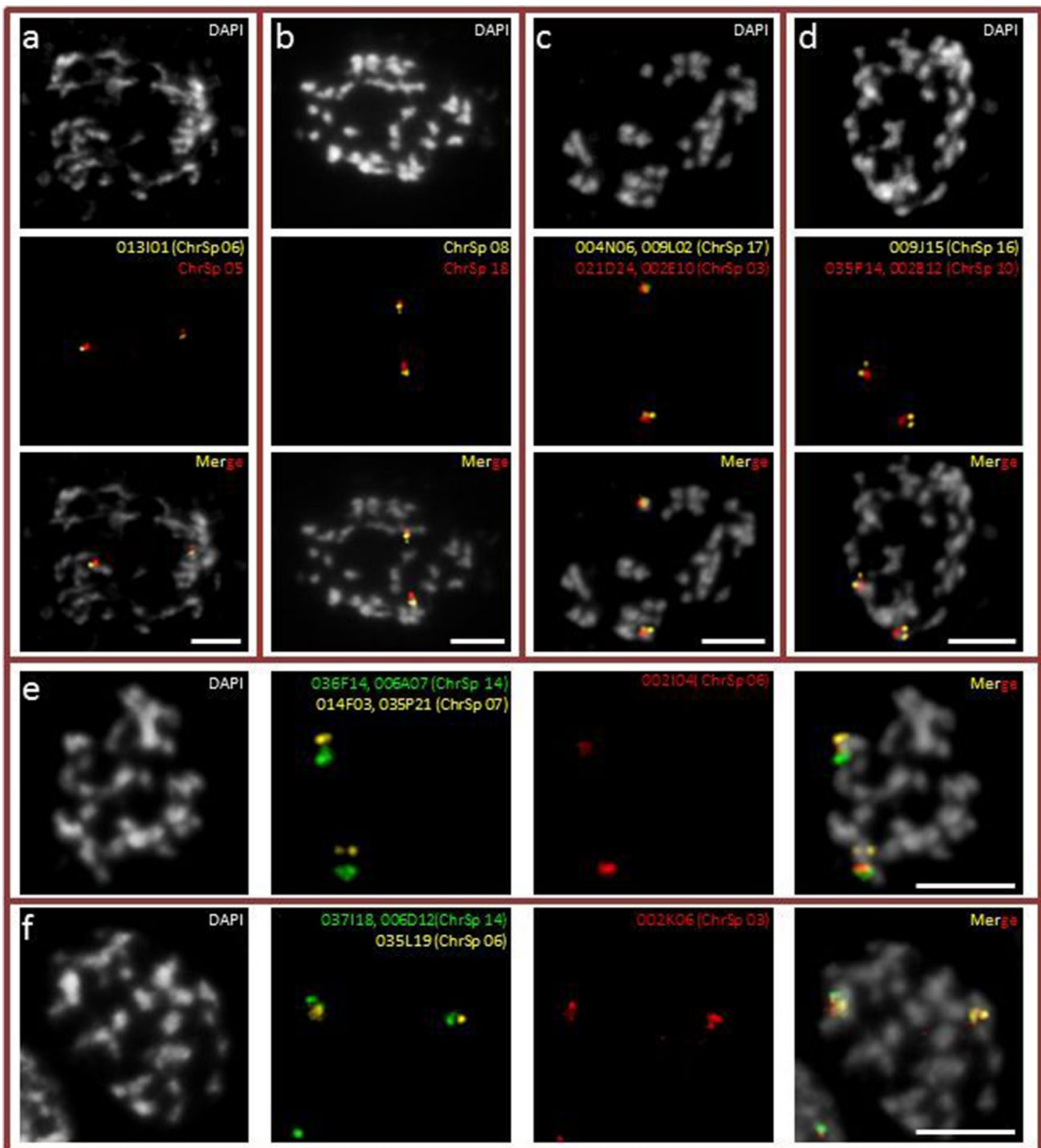

Figure 1. Six linkages due to chromosome rearrangements between S. polyrhiza and S. intermedia (clone 8410) are also present in the sequenced clone 7747. (a) ChrSp05-ChrSp06 =ChrSi06; (b) ChrSp08-

ChrSp18 =ChrSi09; (c) ChrSp03-ChrSp17 =ChrSi04; (d) ChrSp10-ChrSp16 =ChrSi11; (e) ChrSp06-ChrSp07ChrSp14=ChrSi07; (f) ChrSp03-ChrSp06-ChrSp14=ChrSi03. See also Fig. 3. Scale bars $=5 \mu \mathrm{m}$.

BUSCO program v. 3.1.0 ${ }^{36,37}$ with Embryophyta dataset 10 including 1375 genes (mostly from land plants). The hybrid assembly of ON/Illumina reads revealed $96.1 \%$ of the 1375 searched genes (Table 1 ).

Gene prediction. Based on similarity to nine aquatic and non-aquatic angiosperm reference genomes, including two duckweed species, S. polyrhiza $7498 \mathrm{v} 3.1^{25}$ and Lemna minor $5500^{38}$, gene model prediction via Gene Model Mapper-GeMoMa ${ }^{39}$ suggested in total 22,245 (Pacbio, clone 7747) or 21,594 (ON/Illumina, clone 8410) protein/RNA-coding genes, some more than predicted for S. polyrhiza $a^{25}$ (Table S1).

A total number of 16,162 genes of clone $7747,16,493$ of clone 8410 and 11,327 of S. polyrhiza clone 9509 are coinciding with eggNOGs (Non-supervised Orthologous Groups). Comparing the proportion of eggNOG functional categories between the genomes of the two Spirodela species, the differences were $<1 \%$. Only the category 'Energy production and conversion', is overrepresented in S. intermedia clone 7747 (4.2 versus $3.1 \%$ in clone 8410 and $2.6 \%$ in S. polyrhiza) and the category 'Replication, recombination and repair' in clone 8410 (5.3\% versus $3.2 \%$ in clone 7747 and $2.3 \%$ in S. polyrhiza) (Fig. S2, Table S2). 


\begin{tabular}{|c|c|c|}
\hline Sequencing technique & PacBio (clone 7747) & ON/Illumina (clone 8410) \\
\hline \multicolumn{3}{|l|}{ Input data } \\
\hline Read coverage $^{\mathrm{a}}$ & $37.6 \times$ & $191 \times$ \\
\hline Chromosome number & \multicolumn{2}{|l|}{18} \\
\hline Genome physical size ${ }^{\mathrm{b}}$ & \multicolumn{2}{|l|}{$\sim 160 \mathrm{Mbp}$} \\
\hline \multicolumn{3}{|l|}{ Assembly statistics and gene prediction } \\
\hline Assembly length $(\mathrm{Mbp})$ & 147.6 & 136.6 \\
\hline Number of pseudomolecules & \multicolumn{2}{|l|}{18} \\
\hline Number of contigs & 584 & 86 \\
\hline Number of scaffolds & 420 & 70 \\
\hline Number of assigned scaffolds & $\begin{array}{l}18 \text { pseudomolecules (featuring } 63 \text { contigs) } \sim 131.4 \mathrm{Mbp} \text { total } \\
\text { length }\end{array}$ & 18 pseudomolecules (featuring 34 contigs) $\sim 134 \mathrm{Mbp}$ total length \\
\hline Largest scaffold length (Mbp) & $12.5(\mathrm{Si09)}$ & $13.4(\mathrm{Si09)}$ \\
\hline N50 scaffold length (Mbp) & 8.3 & 9.25 \\
\hline $\mathrm{G}+\mathrm{C}$ content $(\%)$ & 41.6 & 42.0 \\
\hline Number of predicted gene models & 22,245 & 21,594 \\
\hline \multicolumn{3}{|c|}{ Completeness of gene prediction (BUSCO) $)^{\mathfrak{c}}$} \\
\hline Complete genes $(\mathrm{C})$ & $1097(79.8 \%)$ & $1280(93.1 \%)$ \\
\hline Complete and single-copy (S) & $1085(78.9 \%)$ & $1266(92.1 \%)$ \\
\hline Complete and duplicated (D) & $12(0.9 \%)$ & $14(1.0 \%)$ \\
\hline Fragmented genes $(\mathrm{F})$ & $131(9.5 \%)$ & $41(3.0 \%)$ \\
\hline Missing genes $(\mathrm{M})$ & $147(10.7 \%)$ & $54(3.9 \%)$ \\
\hline Total number of BUSCO genes used & \multicolumn{2}{|l|}{1375} \\
\hline
\end{tabular}

Table 1. Assembly statistics of $S$. intermedia clones. a Based on genome size measurements by FCM.

${ }^{\mathrm{b}}$ Measured by FCM. ${ }^{\mathrm{c}}$ Reference database odb10.
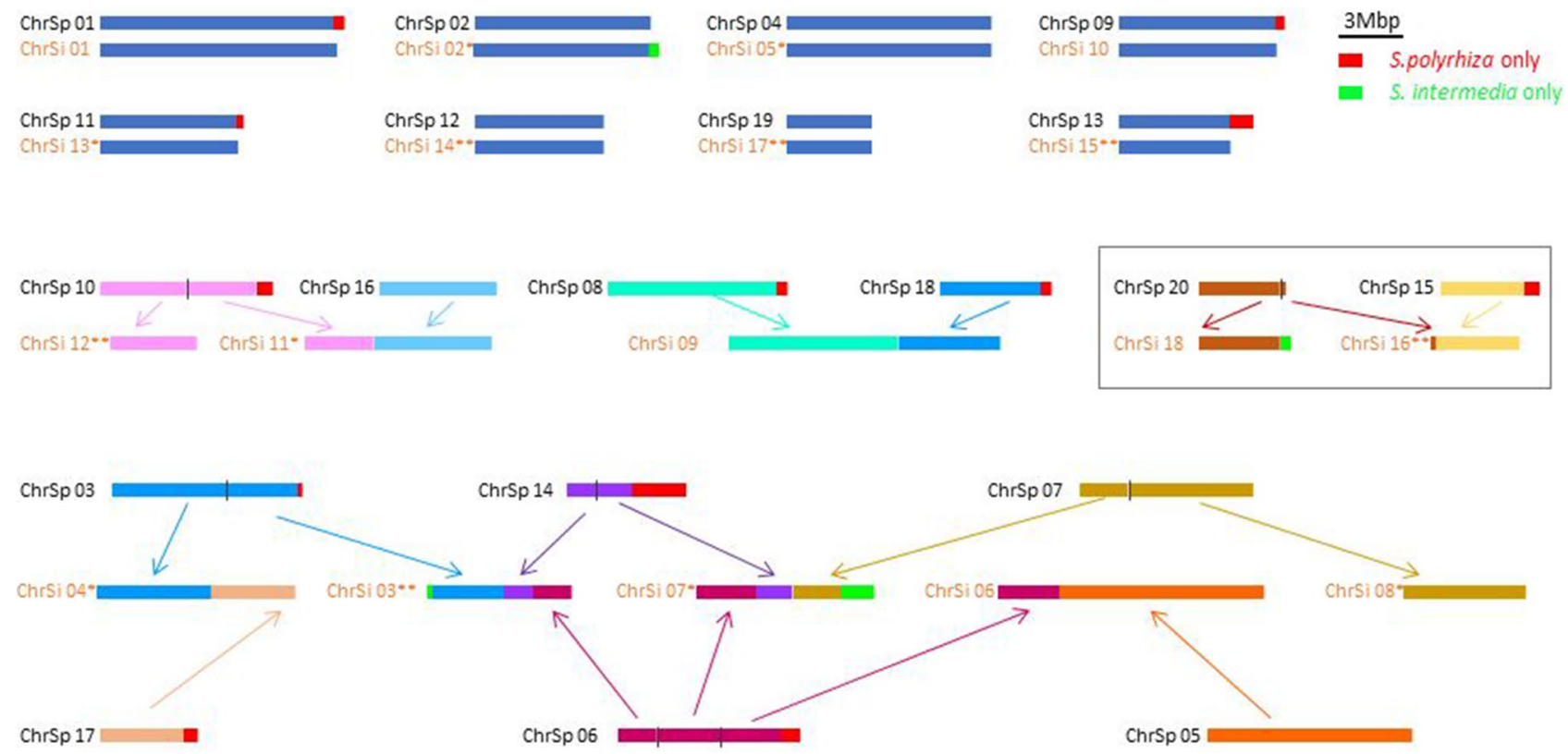

Figure 2. Rearrangements between S. polyrhiza $(\mathrm{n}=20)$ and S. intermedia $(\mathrm{n}=18)$, confirmed for chromosomes of clones 8410 and 7747. Enframed: newly found rearrangement. Red boxes: present only in S. polyrhiza; green boxes: sequences present only in S. intermedia. Scale bar =3 Mbp (based on PacBio assembly for clone 7747). Enumeration is as in Hoang \& Schubert $(2017)^{29}$ and in Table S3 (Short sequences present in S. p., but not chromosomally assigned in S. i. correspond to "SiUn" and are not considered). $13 \mathrm{ChrSi}$ of the 7747 assembly show telomeric sequences at one $\left(^{*}\right)$ or both ends $\left({ }^{*}\right)$, while all pseudomolecules of the 8410 assembly show them at both ends. 


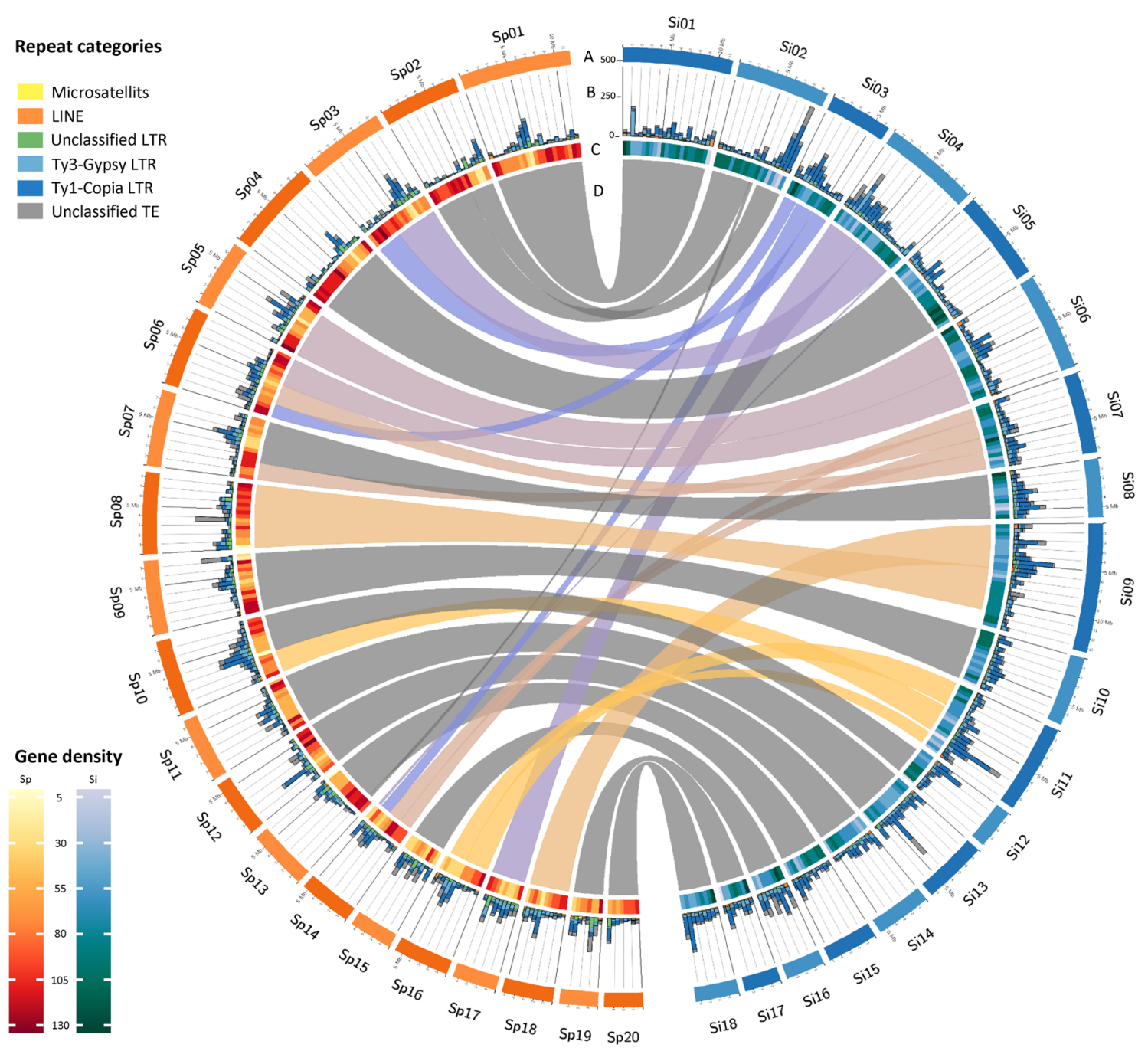

Figure 3. Circos plot of genomes of S. polyrhiza 9505 (orange) and S. intermedia 8410 (blue). (A) tracks representing the size of the pseudomolecules with a corresponding scale in $1 \mathrm{Mbp}$ steps, with highlights every $5 \mathrm{Mbps}$, (B) total length of repeat features (in kbps) (C) gene density and (D) pairwise sequence synteny. The synteny link between gi|13 and Si02 is based on an error in the ON assembly for clone 9509 (see Fig. 5). The region in question actually belongs to gi| 2 as does the remaining part of Si02. Gene and repeat density are plotted in $0.5 \mathrm{Mbps}$ bins. Data for S. polyrhiza 9505 are from Michael et al. $2017^{25}$.

New linkages in S. intermedia as revealed by genome assembly and FISH. The PacBio assembly for clone 7747 indicated a new rearrangement involving ChrSp20. This chromosome did not form the entire ChrSil8 as reported ${ }^{29}$, instead it is split into two parts, the largest part corresponding to ChrSil8 and two rather small regions (between ChrSp20 3.72-3.78 and 3.80-3.96 Mbp) transferred to ChrSil6 (42,000-175,000 bp) (Table S3). Therefore, the previously not tested BAC $013 \mathrm{O} 04$ belonging $\mathrm{ChrSp} 20$ was selected for mcFISH experiments. The presence of this BAC sequence in S. polyrhiza ChrSp20 was confirmed by FISH (Fig. 4a). FISH results on S. intermedia chromosomes (clone 8410: Fig. 4b, and clone 7747: Fig. 4c) showed that BAC $013 \mathrm{O} 04$ (yellow) labeled the same chromosome (ChrSi16) as the BACs belonging to ChrSp15 (red), while the remaining part of ChrSp20 (green) labeled another chromosome pair (ChrSi18). This result confirmed that ChrSp20 became split and a very small part became translocated to ChrSp15, forming ChrSil6 (Fig. 2), as inferred by PacBio assembly. Because only $76 \mathrm{kbp}$ of the BAC $013 \mathrm{O} 04$ sequence appeared in the ON/Illumina assembly of $\mathrm{ChrSi16}$, this link is not visible in Fig. 3 where the entire chromosome ChrSp20 is represented by the S. intermedia chromosome ChrSil8, based on the assembly results for clone 8410 .

Furthermore, a piece of ChrSp13 (0.46-0.68 Mpbs), according to the ON assembly for ChrSp13 of S. polyrhiza clone 9509 , became integrated into $\mathrm{ChrSi02}(5.43-5.66 \mathrm{Mbps})$ as suggested by the assemblies for both $S$. intermedia clones (for clone 8410 see Fig. 3). However, three BACs from this region (029A10, 028I16 and 037J09, together 649,507 bp) used as FISH probe, labeled ChrSp02 of S. polyrhiza clones 7498 and 9509 (Fig. 5) and appear on ChrSp02 also in the Bionano map (CP019095.1) of S. polyrhiza clone $9509^{25}$ as well as in pseudomolecule 1 (corresponding ChrSp02) of S. polyrhiza clone $7498^{23}$ (Table S4). This uncovers a hitherto overlooked error in the ON assembly for S. polyrhiza clone $9509^{24}$. Another new small region, for which no BAC is available, 


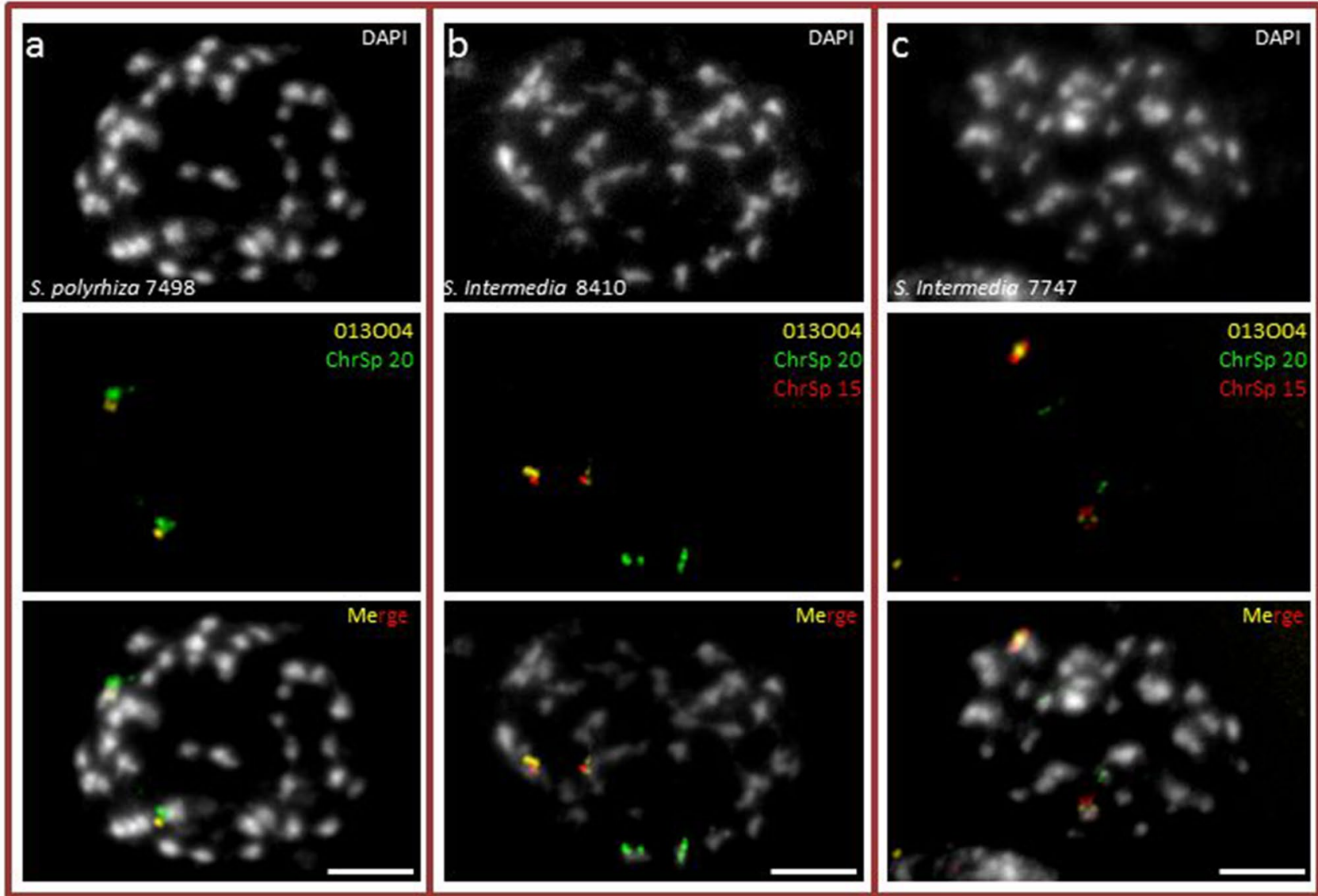

Figure 4. New rearrangement between ChrSp 20 and ChrSp 15 in S. intermedia (a) The newly tested BAC $013 \mathrm{O} 04$ (yellow) belongs to ChrSp 20 (green) in S. polyrhiza; (b,c) BAC $013 \mathrm{O} 04$ was translocated to ChrSp 15 (red) forming ChrSi 16 in S. intermedia clones 8410 and 7747. Scale bars $=5 \mu \mathrm{m}$.

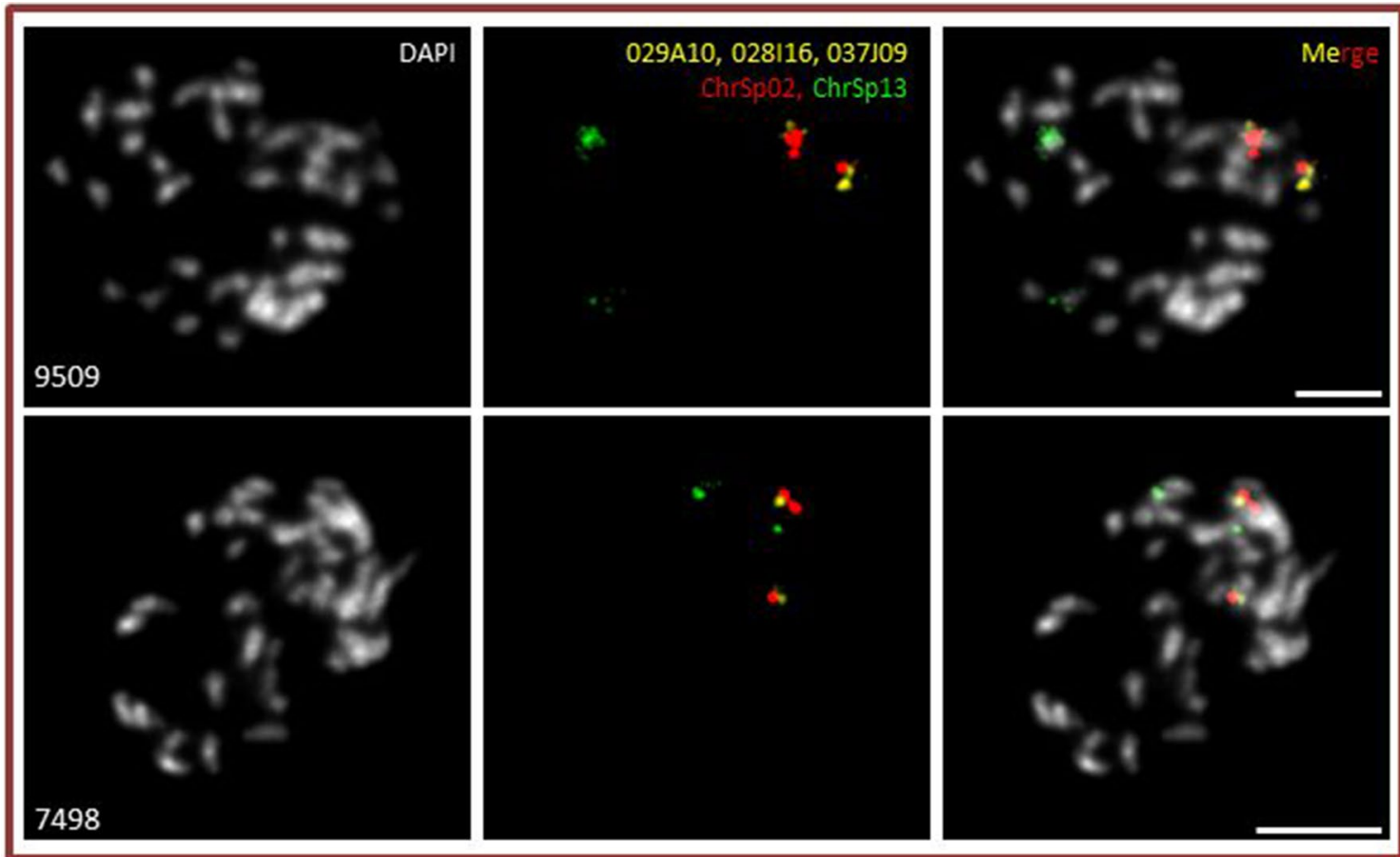

Figure 5. Evidence for ON mis-assembly of chromosome 13 of S. polyrhiza 9509 . The newly tested BACs 029A10, $028 \mathrm{I} 16$ and 037J09 (yellow) belong to ChrSp 02 (red) of S. polyrhiza clone 9509 (upper panel) and clone 7498 (lower panel), not to ChrSp13. Scale bars $=5 \mu \mathrm{m}$. 


\begin{tabular}{|l|l|l|l|}
\hline \multirow{2}{*}{ Repeat } & $\mathbf{8 4 1 0}$ & $\mathbf{8 4 1 0}$ & $\mathbf{7 7 4 7}$ \\
\cline { 2 - 4 } Ty3/gypsy & Illumina reads & Assembly & Assembly \\
\hline Athila & 7.44 & 7.23 & 8.57 \\
\hline CRM & 1.09 & 1.02 & 1.13 \\
\hline Reina & 0.36 & 0.32 & 0.30 \\
\hline Galadriel & 0.05 & 0.04 & 0.05 \\
\hline Tekay & 0.02 & 0.00 & 0.00 \\
\hline Ty1/copia & \multicolumn{4}{|l}{} \\
\hline Ale & 1.87 & 1.78 & 1.79 \\
\hline Ivana & 0.94 & 0.91 & 0.97 \\
\hline Tork & 0.69 & 0.71 & 0.70 \\
\hline Ikeros & 0.40 & 0.37 & 0.39 \\
\hline Unclassified & 0.23 & 0.21 & 0.27 \\
\hline LTR unclass & 1.01 & 0.93 & 1.08 \\
\hline LINE & 0.37 & 0.42 & 0.49 \\
\hline Satellite & 0.17 & 0.06 & 0.09 \\
\hline Microsat. (GA)n & 0.67 & 1.02 & 0.98 \\
\hline rDNA & 0.47 & 0.34 & 0.06 \\
\hline Unclassified & 4.21 & 3.11 & 3.35 \\
\hline Total & 20.00 & 18.49 & 20.22 \\
\hline RepeatScout & n.a & 23.11 & 25.58 \\
\hline
\end{tabular}

Table 2. Repeat proportions [\%] estimated for unassembled sequence reads and genome assemblies of $S$. intermedia clones 8410 and 7747.

became transferred from ChrSp14 (4.9-5.4 Mbps) to ChrSi04 (9.5-9.9 Mbps) and appeared in both assemblies (for clone 8410 see Fig. 3).

Although only one BAC from ChrSp16 was tested previously ${ }^{29}$, the assemblies from PacBio and ON reads suggests that the entire ChrSp16 is included in S. intermedia ChrSi11 together with a part of ChrSp10 (Figs. 2, 3).

Types, abundance and distribution of repetitive elements. Characterization of $S$. intermedia repetitive sequences was first performed by analyzing unassembled Illumina reads from the clone 8410 using the RepeatExplorer pipeline $e^{40}$. This analysis served as a control for repeat quantification in the assembled pseudomolecules that may be biased due to the exclusion of satellite DNA or other repeats that are hard to assemble. In addition, the RepeatExplorer output was used to compile a reference database of $S$. intermedia repeats that was used to annotate the genome assemblies.

The RepeatExplorer analysis of 2.4 million paired-end Illumina reads $(2.25 \times$ genome coverage $)$ revealed relatively small proportions of highly and moderately repeated sequences in the $S$. intermedia genome (Table 2). The repeats accounted for $20 \%$ of the genome, with LTR-retrotransposons representing the most abundant repeat class (14.1\% of the genome, with Ty3/gypsy to Ty1/copia ratio of 2.17). Other repeats including LINEs, rDNA and satellite DNA made up only minor genome proportions.

Repeat annotations of the genome assemblies using the RepeatExplorer reference database resulted in $18.5 \%$ and $20.2 \%$ of the 8410 and 7747 sequences marked as repetitive, respectively. These proportions correspond to the estimate obtained for unassembled reads (20.0\%), suggesting that repeats were not significantly depleted in the final assemblies. Inspection of individual repeat categories revealed that partial depletion resulting in smaller proportions in the assembled genomes occurred for satellite repeats in both clones and for rDNA in the clone 7747 (Table 2). To obtain an alternative estimate of repeat proportions, we also analyzed both assemblies using RepeatScout ${ }^{41}$ that performs de-novo identification of repetitive elements based on the high frequency k-mers. This program estimated total repeat proportions of $23.1 \%$ (8410) and $25.6 \%$ (7747), most likely due to its better sensitivity for low-copy repeats (Table 2).

An interesting observation was the relatively high abundance of simple-sequence repeats, especially the microsatellite motif $(\mathrm{AG})_{\mathrm{n}}$, that was revealed by Tandem Repeats Finder ${ }^{42}$ analysis within the assembled genomes and the Illumina reads. About 37,000 loci of $(\mathrm{AG})_{\mathrm{n}}$ with an average length of $38 \mathrm{bp}$ were detected in 8410 , making up $1 \%$ of the assembly (the same proportion and characteristics were found for $(A G)_{n}$ in the 7747 assembly). These dispersed simple repeat loci appeared in the $S$. intermedia genome with an average frequency of one per $3.7 \mathrm{~kb}$.

Characterization of rDNA loci. Chromosome analysis of S. intermedia by FISH using 5 S and (18S + 26S) rDNA probes, detected one major locus of $5 \mathrm{~S}$ rDNA on ChrSi15 (corresponding to ChrSp13) (Fig. S3a) and a major locus of $45 \mathrm{~S}$ rDNA on ChrSi01 (corresponding to ChrSp01) (Fig. S3b). The chromosome-scale assembly and ON reads for Si8410 confirmed the presence of a 5S rDNA locus on ChrSi15, and revealed an additional 
a

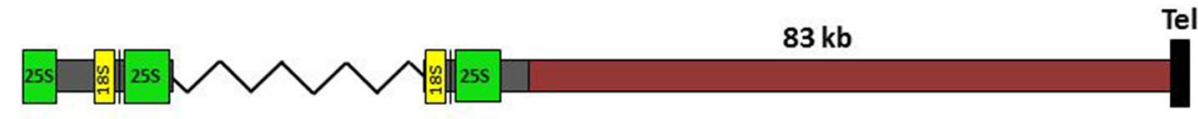

45 S rDNA: SiChr-1

45S rDNA: SiChr-6

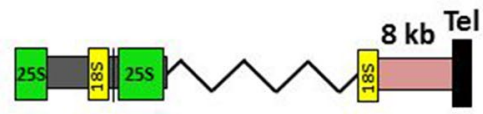

b

Type 1

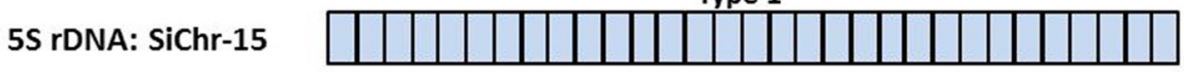

Type 2

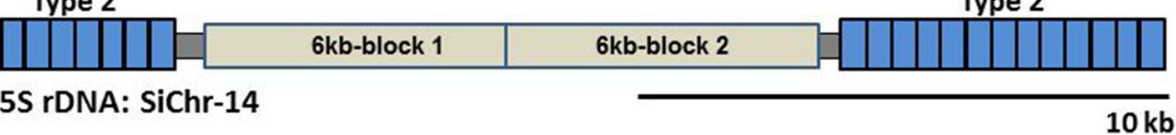

Figure 6. Schematic representation of rDNA loci in S. intermedia 8410. (a) Depiction of 45S rDNA loci at chromosomes ChrSi01 and ChrSi06; Tel = telomere. (b) Depiction of 5S rDNA loci at chromosome ChrSi15, and ChrSil4. The ChrSi15 locus contains 31 5S rDNA units with NTS of type-1; the ChrSil4 locus is composed of two clusters of 7 and $135 \mathrm{~S}$ rDNA units of type-2 NTS, separated by a doubled $6 \mathrm{~kb}$ sequence of unknown function.

locus on ChrSi14. The extra-long ON reads showed that the locus on ChrSi15 contains a cluster of thirty-one 5S rDNA repeats, whereas the locus on ChrSil4 is composed of seven and $135 \mathrm{~S}$ rDNA repeats interrupted by two $6 \mathrm{~kb}$ long repeats of another sequence (Fig. 6b). The chromosome assembly, based on PacBio sequences, showed similar arrangement of 5S rDNA in Si7747 with slightly different copy numbers in each of the loci. Quantitative PCR estimation of 5S rDNA copy number in Si7747 and Si8410 genomes supports this data with $57 \pm 10$ copies of $5 \mathrm{~S}$ rRNA genes for Si8410 and, $70 \pm 20$ copies for Si7747. Sequence alignments suggested that the 5SrDNA units of the two loci contain slightly different non-transcribed spacers (NTS). This was confirmed by sequencing of the 5S rDNA repeats, amplified from genomic DNA of clones 8410 and 7747. Analysis of individual 5S rDNA clones of Si8410 and Si7747 identified the 119 bp long 5S rRNA genes with $100 \%$ identity to the previously sequenced 5S rRNA gene of S. polyrhiza $a^{43}$, and two variants of the NTS for each ecotype (Fig. S4), with variant 8410-1 corresponding to 5S rDNA locus on chromosome ChrSil5 and the variant 8410-2 corresponding to the locus on chromosome ChrSil4 (Fig. 6a).

In agreement with the FISH data, the genome assembly revealed a $45 \mathrm{~S}$ rDNA locus in a distal position on ChrSi01 for both clones, Si7747 and Si8410. Extra-long ON reads confirmed this locus for Si8410, about $83 \mathrm{~kb}$ upstream of one $\mathrm{ChrSi01}$ chromosome telomere. Moreover, ON reads revealed an additional cluster of $45 \mathrm{~S}$ rDNA repeats about $8 \mathrm{~kb}$ upstream the telomere on ChrSi06. Unlike the situation with $5 \mathrm{~S} \mathrm{rDNA}$ loci, there are no readthrough sequences containing the whole $45 \mathrm{~S}$ rDNA loci. Therefore, it is not possible to determine exactly how many of the $93 \pm 2545$ rDNA copies estimated by qPCR for Si8410, are located in the minor locus.

\section{Discussion}

Chromosome rearrangements between the two Spirodela species. Previously, chromosome homeology and rearrangements between $S$. polyrhiza and $S$. intermedia were uncovered by comparative serial multicolor cross-hybridization to the $S$. intermedia clone $8410^{29}$. Here, we applied the same approach, using informative BACs out of the 96 ones anchored in the $S$. polyrhiza genome, and found the same chromosomal rearrangements in S. intermedia clone 7747 as reported for clone $8410^{29}$. Reiterative rounds of genome assembly and validation by FISH revealed a new linkage compared to the $S$. polyrhiza genome, in addition to the eight translocations detected previously ${ }^{29}$. This new linkage involves ChrSil6, which received small parts from one end of ChrSp20 (Fig. 2). A small piece, apparently transferred from one end of ChrSp13 to ChrSi02, turned out to be an error within the previous ON assembly for ChrSp13 of clone $9509^{24}$. Another small region, transferred from ChrSp14 to $\mathrm{ChrSi04}$ was not studied by FISH, but it appeared in both assemblies. The S. intermedia chromosomes $\mathrm{ChrSi03,04,06,07,09}$ and 11, previously found to be involved in evolutionary rearrangements, correspond in total to $10 \mathrm{~S}$. polyrhiza chromosomes (Figs. 1, 2, $3 \mathrm{and}^{29}$ ). Now, at least two more rearrangements (in addition to the previously postulated one inversion and eight translocations) have to be assumed if the $S$. polyrhiza karyotype is more similar to that of the ancestor. Alternatively, eight (instead of six) translocations (involving ChrSi03, 04, 06, 07, 08, 11, 12, 16, 18), and one fission $(\mathrm{ChrSi09})^{29}$ were required if the S. intermedia karyotype is the more ancestral one. Additionally, we found a positional change of the smaller $5 \mathrm{~S}$ rDNA locus to ChrSil4 (instead of ChrSi07 which harbors sequences adjacent to the minor $5 \mathrm{~S}$ rDNA locus on ChrSp06).

The increased number of rearrangements refined our knowledge about karyotype evolution between S. polyrhiza and S. intermedia and made the corresponding genome assembly for both S. intermedia clones robust by 
independent confirmation. These results demonstrate the benefit of our novel approach, combining comparative cytogenomics and hybrid sequencing technologies, especially for species for which genetic maps are not available.

Genome assembly for both S. intermedia clones. The assembly of PacBio reads for clone 7747 is more fragmented than the assembly of ON/Illumina reads for clone 8410. It displays a higher percentage of ambiguous bases $(0.003 \%)$ and a lower coverage (Table 1). Several draft contigs representing contamination from plastids, mitochondria, and a virus (Human alpha herpesvirus 1 strain) were detected which show largely increased coverages of 2925-, 17,126- and 73-fold respectively. These overrepresented sequences caused a diminished overall coverage in both assemblies. Canu performs best with more than 50X coverage, while lower values might decrease the contiguity of an assembly ${ }^{32}$.

Comparing read length N50 of the two S. intermedia clones, the ON library of clone $8410(16,322 \mathrm{~kb})$ yielded a larger amount of long reads than clone 7747 with an N50 of $9879 \mathrm{~kb}$. As a consequence, longer repeats may not be sequenced completely, leading to a more fragmented assembly ${ }^{44}$, as in case of clone 7747 .

In summary, the PacBio read assembly of S.intermedia 7747 provides continuous pseudomolecules but these might comprise ambiguous and low coverage nucleotide stretches, and possibly missing sequences, as described for other plant species ${ }^{45}$. Despite the lower library quality for clone 7747, the non-hybrid PacBio assembly shows (as expected ${ }^{46}$ ) good results for the scaffold N50 (8.3 Mbp similar to 9.25 of the ON/Illumina assembly, Table 1), depicting the synergy of cytogenetics and long read sequencing technologies.

The distribution of functional eggNOG groups within the S. intermedia clones and S. polyrhiza is largely balanced, indicating a high similarity in overall functional gene content between both species which amounts with 20,000 genes, a rather low number compared to most land plants. The BUSCO results reflect the increased fragmentation of the 7747 assembly by an increased number of fragmented and missing genes ( $20 \%$ in total). With $3.0 \%$ of fragmented and $3.9 \%$ of missing candidate genes, the ON based assembly of clone 8410 exhibits a good representation of the expected gene content. It has to be noted, that the embryophyta set of orthologue sequences (mostly from land plants) might not be optimal for aquatic plants, because unnecessary genes might have been lost during evolutionary organ reduction and aquatic life style of duckweeds ${ }^{21}$.

Repetitive elements. S. intermedia revealed a rather low proportion of detected repeats $(\sim 25 \%)$, in comparison with other genomes of similar size, such as that of $A$. thaliana $\left(157 \mathrm{Mbp} / 1 \mathrm{C} ; 32 \%{ }^{47}\right)$, or the even smaller genome of Genlisea nigrocaulis $(86 \mathrm{Mbp} / 1 \mathrm{C})$ with $15.9 \%$ of total repeats ${ }^{48}$. Similar repeat proportions were reported by Wang et al. (2014) $)^{21}$ for S. polyrhiza, however, later Michael et al. (2017) ${ }^{25}$ reported a higher proportion of mobile elements (25\%) for that species. Possible explanations might be: (1) There is a difference in repeat content between S. polyrhiza and S. intermedia; this seems less likely, because of the close relationship and same genome size of both species. (2) The assembly by Michael et al. ${ }^{25}$ is more complete; this is probably not the case, because we found a lower content of mobile elements also in unassembled reads. (3) Their methods including structure-based detection revealed low copy elements (one or a few copies/C) which are not captured by our clustering and similarity-based searches. (4) Michael et al. ${ }^{25}$ described a ratio of soloLTRs to complete elements of 8 to 1 . Because our tools did not determine soloLTRs, the repeat content for S. intermedia might be underestimated. A high proportion of soloLTRs suggests that the genome size of Spirodela decreased by deletion-biased DNA double-strand repair ${ }^{49}$ via the single-strand annealing pathway since its separation from the other duckweed lineages. Remarkable is the high abundance of SSR repeats, especially that of dispersed (AG) ${ }_{\mathrm{n}}$ microsatellite arrays, which in rice contribute to regulate gene expression by binding transcription factors ${ }^{50}$. Such arrays were reported also for $S$. polyrhiza, where they "severely impeded elongation during sequence assembly"21.

The unusually low copy number of both $45 \mathrm{~S}$ and $5 \mathrm{~S}$ rDNA repeats revealed in the genome of $S$. polyrhiza ${ }^{24,25}$, inspired curiosity about the number and arrangement of those genes in related S. intermedia. The qPCR-based estimation showed $~ 93$ copies of the 25S rRNA genes for both Si8410 and Si7747 genomes, a number very close to the estimate for S. polyrhiza ${ }^{25}$. Unlike S. polyrhiza, where the $45 \mathrm{~S}$ rDNA was shown to locate in a single chromosome locus, our deep coverage ON sequencing of Si8410 revealed at least two 45S rDNA loci, located at $\sim 83 \mathrm{~kb}$ upstream of the telomere at SiChr01, and at $\sim 8 \mathrm{~kb}$ upstream of the telomere at SiChr06. The fact that FISH revealed only one signal on SiChr01, suggests that this location is the major 45S rDNA locus with the majority of the $\sim 93$ gene repeats. However, the exact $45 \mathrm{~S}$ rDNA copy number distribution between the two loci remains unclear, because no read-through sequences are available. For the $5 \mathrm{~S} \mathrm{rDNA}$, a range of generated $\mathrm{ON}$ read-through sequences, revealed two distinct loci, one containing a cluster of 31 repeats on SiChr 15 which has been also visualized by FISH, and a second split locus on SiChr14 containing two clusters with 7 and 13 repeats, separated by $\sim 13 \mathrm{~kb}$ long region of non-rDNA sequence (Fig. 6b). While the general arrangement of the $5 \mathrm{~S}$ rRNA genes in $S$. intermedia resembles that of the $S$. polyrhiza, the total $5 S$ rDNA copy number in $S$. intermedia is even lower, 51 vs 73 in $S$. polyrhiza ${ }^{24}$. This is the smallest number reported so far for any plant species. Usually, the copy number of 5S RNA genes in land plants varies from 2000 to $75,000^{51}$. Therefore, our findings for S. polyrhiza and $S$. intermedia proclaim that the small copy number of rDNA is a unique phenomenon of the genus Spirodela.

\section{Material and methods}

Plant material. Spirodela intermedia W. Koch (accessions 8410 from Panama City and 7747 from Lima, Peru) were obtained from Elias Landolt's collection via Klaus Appenroth, University of Jena and Rutgers Duckweed Stock Cooperative (New Jersey, USA). The fronds were grown in liquid nutrient medium ${ }^{52}$ under $16 \mathrm{~h}$ white light of $100 \mu \mathrm{mol} \mathrm{m} \mathrm{m}^{-2} \mathrm{~s}^{-1}$ at $24^{\circ} \mathrm{C}$.

Genomic DNA isolation. Genomic DNA of S. intermedia (clone 7747) was extracted from fresh fronds using the DNeasy Plant Mini Kit (Qiagen) for PacBio sequencing. High molecular weight DNA of S. intermedia 
(clone 8410) was isolated for Oxford Nanopore and Illumina sequencing as follows: the plants were kept three days in the darkness. After harvesting, $10 \mathrm{~g}$ of the fronds were used for DNA isolation from purified nuclei according to the protocol of Vondrak et al. ${ }^{53}$, with two minor modifications: the centrifugation of nuclei was performed at $650 \times \mathrm{g}$ instead of $200 \times \mathrm{g}$ due to their small size, and $2 \times \mathrm{CTAB}$ isolation buffer was supplemented with 2\% PVP-360 (polyvinylpyrrolidone, avg. molecular weight 360,000).

Genome sequencing. PacBio. After shearing of genomic DNA (S. intermedia clone 7747), a size-selected $20 \mathrm{~kb}$ library was sequenced on the Pacific Biosciences RS II platform (GATC Biotech, Konstanz, Germany) combining the P6-C4 polymerase-chemistry and $240 \mathrm{~min}$ of movie duration. Two rounds of sequencing resulted in $149 \mathrm{~Gb}$ of raw read data.

Oxford nanopore. The sequencing libraries were prepared from $3 \mu \mathrm{g}$ of purified HMW DNA using a Ligation Sequencing Kit SQK-LSK109 (Oxford Nanopore Technologies) as described by Vondrak et al. ${ }^{53}$. Briefly, the DNA was treated with $2 \mu$ of NEBNext FFPE DNA Repair Mix and $3 \mu$ l of NEBNext Ultra II End-prep enzyme mix in a $60 \mu \mathrm{l}$ volume that also included $3.5 \mu \mathrm{l}$ of FFPE and $3.5 \mu \mathrm{l}$ of End-prep reaction buffers (New England Biolabs). The reaction was performed at $20^{\circ} \mathrm{C}$ for $5 \mathrm{~min}$ and $65^{\circ} \mathrm{C}$ for $5 \mathrm{~min}$. Then, the DNA was purified using a $0.4 \times$ volume of AMPure XP beads (Beckman Coulter). Because long DNA fragments caused clumping of the beads and were difficult to detach, the elution was performed with $3 \mathrm{mM}$ TRIS- $\mathrm{HCl}$ (pH 8.5) and was extended up to $40 \mathrm{~min}$. Subsequent steps including adapter ligation using NEBNext Quick T4 DNA Ligase and library preparation for sequencing were performed as recommended. The whole library was loaded onto FLO-MIN106 R9.4 flow cell and sequenced on MinION instrument until the number of active pores dropped below 40 (21$24 \mathrm{~h}$ ). Basecalling of the raw reads was done using Albacore 2.3.3 (Oxford Nanopore Technologies).

Illumina. Illumina sequencing was performed by Admera Health, LLC (South Plainfield, NJ, USA) using a KAPA DNA Library kit (Roche) and resulted in 130 million paired-end reads $(2 \times 150 \mathrm{nt})$.

Sequence assembly and pseudomolecule construction. After an initial filtering for potential bacterial contamination (blastn against microbial NCBI refseq database from Aug 2017) and minimum read length (500 nucleotides), PacBio reads were assembled using the Canu pipeline v. $1.5^{32}$ (options: 'genomeSize $=160 \mathrm{~m}$, correctedErrorRate $=0.105^{\prime}$ ) consisting of the following steps:

(1) Trimming, error correction and contig construction: Reads were corrected and trimmed by comparing overlaps. A minimum length of 500 nucleotides and a maximum error rate of $10.5 \%$ was chosen for extending a contig. Only reads consisting of more than 1000 nucleotides in length were considered in this step. Afterwards, the corrected reads were trimmed to improve overall read quality by using overlap information to detect high confidence regions. Contigs of insufficient read coverage and/or containing 'noisy' sequence were categorized as 'unsupported regions' and divided at weak sequence positions into subcontigs with higher support.

After further contig construction on the basis of overlaps, a consensus sequence was built by removing the remaining sequencing errors to raise the overall assembly quality.

(2) Scaffolding and gap filling

In a first round of scaffolding, the two genomes of the sister species S. polyrhiza (from clones 9505 and $7498)^{23,25}$ were used as references for Mauve Genome Aligner v20150522 $2^{33}$ to order contigs. Scaffolding was performed by SSPACE-Longread v.1-1 ${ }^{34}$ (default options). The resulting scaffold assembly was used for the super-scaffolding approach. For this aim, contigs were assigned to 18 putative pseudomolecules (corresponding to the $18 \mathrm{~S}$. intermedia chromosomes) using the information of cross-FISH of $93 \mathrm{~S}$. polyrhiza BACs on the chromosomes of S. intermedia clone $8410^{29}$. New cytogenetic probes using BACs from the genomic regions of interest were designed for FISH experiments to approve localization of the contigs within the pseudomolecules and to resolve mis-assemblies. Additionally, bacterial contamination was filtered as described previously.

The quality of both S. intermedia genome assemblies was assessed by the BUSCO program ${ }^{36,37}$ with the Embryophyta odb10 dataset comprising 1375 conserved genes.

The ONT/Illumina assembly for clone 8410 was performed using MaSuRCA ${ }^{54}$. In the first step, super-reads were assembled from 68 million Illumina $2 \times 151 \mathrm{nt}$ paired-end reads (128-fold coverage). Subsequently, 307,111 nanopore reads 10,000-425,377 nt in length (total length 9,963,752,003) and representing a 62-fold genome coverage, were used in scaffolding step. The resulting MaSuRCA assembly consisted of 386 scaffolds (N50 408,333 bp, total length $191,862,084 \mathrm{bp})$. Scaffolds were assigned/ordered into pseudomolecules using the 7747 as reference for a Mauve Genome alignment ${ }^{33}$. Additionally, all the formation of super-scaffolds was accompanied by manual curation steps based on FISH results.

Gene prediction and functional annotation. Gene finding was carried out using Gene Model Mapper (GeMoMa) - a similarity-based gene prediction program ${ }^{39}$ ('GeMoMa-1.6.1.jar CLI GeMoMaPipeline $\mathrm{t}=\mathrm{g}=\mathrm{a}=$ Extractor. $\mathrm{p}=$ true AnnotationFinalizer.r $=$ SIMPLE AnnotationFinalizer. $\mathrm{p}=$ '). Gene models were predicted by combining the predictions based on the genome data of the neighbor species S. polyrhiza and eight additional reference organisms (S. polyrhiza $7498 \mathrm{v} 3.1^{23}$, Lemna minor $5500^{38}$, Arabidopsis thaliana TAIR10, Ananas comosus (Phytozome internal code 321), Brachypodium distachyon (Phytozome internal code 314), 
Nelumbo nucifera 1.1 (GenBank assembly accession: GCF_000365185.1), Panicum hallii v3.1 (GenBank assembly accession: GCF_002211085.1), Oryza sativa IRGSP v1.0.38 (GenBank assembly accession: GCA_001433935.1), Zostera marina v2.1 (GenBank assembly accession: GCA_001185155.1)).

Noncoding RNAs were determined using RNAmmer v1.25 ('rnammer -S euk -m tsu,ssu,lsu -h - $\mathrm{f}^{5}$ ) and tRNAscan-SE-2.056 ('tRNAscan-SE - B -o - b - thread 10'). Predicted tRNAs were filtered for features overlapping with protein coding exons ('bedtools intersect $-\mathrm{b}-\mathrm{a}-\mathrm{wa}-\mathrm{wb}-\mathrm{f} 0.8-\mathrm{r}$ ').

Functional annotations and GO terms were assigned using Interproscan 5 v 5.26-65.0 57 ('interproscan.sh -dp -input -seqtype p -f tsv,html,gff3 -applications TIGRFAM, PfamA, SMART, SUPERFAMILY -pathways -goterms'). COGs (clusters of orthologous groups) were computed using eggnog-mapper ${ }^{58}$ online tool ('Taxonomic background: Viridiplantae, default options') based on eggNOG 4.5 orthology data ${ }^{59}$.

Comparative genomics and visualization. Best bi-directional hits (BBHs) were identified using BLASTN 2.2.31+('-evalue 1e-5 -out outfmt 6'). Additional filtering for length and percent identity was applied afterwards (minimum length: $100 \mathrm{nt}$; minimum identity: 80\%). Annotation of gene features and repetitive elements in S. polyrhiza 9505 was taken from Michael et al., 2017 25 . Both, length of selected repeat features and gene density were computed across a $0.5 \mathrm{Mbp}$ window using the bedops suite ${ }^{60}$.

Feature annotation of each annotated repeats and genes as well as chromosome sizes have been converted to bed format using 'gtf2bed'. From these files the $0.5 \mathrm{Mbp}$ windows across each chromosome have been calculated with bedops -chop. Finally, the counts of gene and repeat features within each chromosome has been determined by bedmap -count.

Synteny was determined using minimap2 ('minimap2 -x') 35 between S. polyrhiza and S. intermedia. 8410. Overlapping intervals have been merged by bedtools ${ }^{61}$ merge (default options). Links shorter than 10.000 have been excluded from the analysis.

To compare the two Spirodela genomes, synteny between pseudomolecules, gene and repeat distribution have been plotted using Circos tool v0.67-1 ${ }^{62}$.

Repeat annotation and analysis. Repeat analysis in unassembled Illumina reads from the clone 8410 was performed using similarity-based clustering implemented in the RepeatExplorer pipeline ${ }^{40}$. The pipeline was run with modified settings in order to increase its sensitivity towards divergent repeats. The similarity search step was done with BLASTN instead of mgblast and the similarity threshold was lowered from 90 to $80 \%$ identity. A total of 2.4 million of $150 \mathrm{nt}$ paired-end reads were used as an input, and repeats were annotated in clusters representing at least $0.005 \%$ of the input reads.

Repeat annotation in the assembled pseudomolecules was performed using several alternative approaches. PROFREP and DANTE modules available at the public RepeatExplorer server (https://repeatexplorer-elixi r.cerit-sc.cz/) were used to annotate repeats based on similarities to the reference database compiled from the repeat clustering analysis described above, and to the REXdb database of conserved protein domains of mobile elements ${ }^{63}$, respectively. Additionally, repetitive sequences in the assemblies were annotated using RepeatScout v. 1.0.5 ${ }^{41}$. First, a library of repeats based on frequent k-mers was created using default parameters. Repeats with a frequency below 10 copies were then removed from the library. The resulting library was then used for annotating genome assemblies using RepeatMasker (https://www.repeatmasker.org).

Identification and quantification of microsatellites (simple sequence repeats) was done by means of Tandem Repeat Finder ${ }^{42}$ using the settings "trf input_file 25780102525 -f" and parsing the output using TRAP ${ }^{64}$.

Molecular characterization of rDNA. The estimation of $25 \mathrm{~S}$ and $5 \mathrm{~S}$ rRNA gene copies was carried out by qPCR, relating the rates of sample DNA amplification to the standard curve. The standard curve was constructed based on the amplification reads of dilution series of a reference plasmid, containing part of the actin gene (single copy in Spirodela polyrhiza, 9509), a whole PCR amplified 5S rDNA unit of S. polyrhiza and a part of the 25 S gene amplified from genomic DNA of S. polyrhiza, 9509, using primers with internal restriction sites for XbaI and EcoRI. The rDNA copy number was determined in qPCR reactions prepared with the UltraSybr Mixture (CWBio, Taizhou, China), run on the CFX Connect Real-Time detection system (Bio-Rad, Hercules, USA). For quantification of the $25 \mathrm{~S}$ rDNA we used the 5'-TCCCACTGTCCCTGTCTACT and 5'-CCCACT TATCCTACACCTCT primers, and for the $5 \mathrm{~S}$ rDNA the set of primers was: $5^{\prime}$-GGGTGCGATCATACCAGCAC and 5'-GGGTGCAACACGAGGACTTC. The samples and tenfold dilution series of the reference plasmid were assayed in the same run. The quality of products was checked by thermal denaturation cycle. Only the experiments providing a single peak were considered. Three technical replicates were performed for each sample. The obtained data were analyzed using the program BIO-RAD CFX Manager 3.1 (Hercules, USA) and Microsoft Excel 2016 software.

For sequencing, the 5S rDNA genes were amplified from genomic DNA by PCR using 5S rRNA gene-specific primers DW-5S-F: CTTGGGCGAGAGTAGTACTAGG and DW-5S-R: CACGCTTAACTTCGGAGT TCTG, purified by gel electrophoresis and cloned into the vector pMD19 (Takara, Dalian, China). The obtained sequences were analyzed using the "Online Analysis Tools" package (https://molbiol-tools.ca).

Mitotic chromosome preparation, probe preparation and FISH. Spreading of mitotic chromosomes was carried out according to Hoang ${ }^{29}$. $5 \mathrm{~S}$ rDNA, $18 \mathrm{~S}$ and $26 \mathrm{~S}$ rDNA probes were generated from $S$. polyrhiza and from S. intermedia genomic DNA each by using designed primer pairs ${ }^{16,21,65,66}$ as described ${ }^{24}$. Ribosomal DNA, A. thaliana type telomere and S. polyrhiza BAC probes were labeled with Cy3-dUTP (GE Healthcare Life Science), Alexa Fluor 488-5-dUTP, Texas red-12-dUTP, biotin-dUTP or digoxigenin-dUTP (Life Technologies) and precipitated as described ${ }^{29}$. 
Denaturation of mitotic chromosomes and probes, hybridization, post-hybridization washing and signal detection were carried out according to Lysak et al. ${ }^{67}$. Probe stripping and re-hybridization were done as described $^{29}$.

Microscopy and image processing. Fluorescence microscopy for signal detection followed Cao et al. ${ }^{23}$. The images were processed (brightness and contrast adjustment only), pseudo-colored and merged using Adobe Photoshop software ver. $12 \times 32$ (Adobe Systems).

\section{Data availability}

The genome assemblies of S. intermedia 7747 and 8410 have been deposited at the European Nucleotide Archive (ENA) under PRJEB35514 and PRJEB35634, respectively. Raw reads can be obtained from EBI ENA using accession numbers PRJEB33624 (PacBio, S. intermedia 7747), ERR3829756 (Illumina, S. intermedia 8410), and ERR3957957-ERR3957958 (Oxford Nanopore, S. intermedia 8410).

Received: 2 July 2020; Accepted: 13 October 2020

Published online: 05 November 2020

\section{References}

1. Goswami, C., Majumder, A., Misra, A. K. \& Bandyopadhyay, K. Arsenic uptake by Lemna minor in hydroponic system. Int. J. Phytoremediation 16, 1221-1227. https://doi.org/10.1080/15226514.2013.821452 (2014).

2. Tatar, ŞY. \& Öbek, E. Potential of Lemna gibba L. and Lemna minor L. for accumulation of Boron from secondary effluents. Ecol. Eng. 70, 332-336. https://doi.org/10.1016/j.ecoleng.2014.06.033 (2014).

3. Teixeira, S., Vieira, M. N., Espinha Marques, J. \& Pereira, R. Bioremediation of an iron-rich mine effluent by Lemna minor. Int. J. Phytoremediation 16, 1228-1240. https://doi.org/10.1080/15226514.2013.821454 (2014).

4. Verma, R. \& Suthar, S. Synchronized urban wastewater treatment and biomass production using duckweed Lemna gibba L. Ecol. Eng. 64, 337-343. https://doi.org/10.1016/j.ecoleng.2013.12.055 (2014).

5. Fourounjian, P., Fakhoorian, T. \& Cao, X. Importance of duckweeds in basic research and their industrial applications. In The Duckweed Genomes (eds Cao, X. H. et al.) 1-17 (Springer, Berlin, 2020). https://doi.org/10.1007/978-3-030-11045-1_1.

6. Vu, G., Fourounjian, P., Wang, W. \& Cao, X. Future prospects of duckweed research and applications. In The Duckweed Genomes (eds Cao, X. H. et al.) 179-185 (Springer, Berlin, 2020). https://doi.org/10.1007/978-3-030-11045-1_18.

7. Ziegler, P., Sree, K. S. \& Appenroth, K. J. Duckweeds for water remediation and toxicity testing. Toxicol. Environ. Chem. 98, 1127-1154. https://doi.org/10.1080/02772248.2015.1094701 (2016).

8. Ziegler, P., Sree, K. S. \& Appenroth, K. J. The uses of duckweed in relation to water remediation. Desalination \& Water Treatment, 63, 327-342 (2017). In 5th International Conference on Environmental Management, Engineering, Planning and Economics, Vol. 63, 327-342 (Balaban Publishers - Desalination Publications, Mykonos, Greece, 2017).

9. Ziegler, P., Adelmann, K., Zimmer, S., Schmidt, C. \& Appenroth, K. J. Relative in vitro growth rates of duckweeds (Lemnaceae) -the most rapidly growing higher plants. Plant Biol. 17(Suppl 1), 33-41. https://doi.org/10.1111/plb.12184 (2015).

10. Appenroth, K.-J. et al. Nutritional value of the duckweed species of the genus Wolffia (Lemnaceae) as human food. Front. Chem. 6, 483. https://doi.org/10.3389/fchem.2018.00483 (2018).

11. Sońta, M., Rekiel, A. \& Batorska, M. Use of duckweed (Lemna L.) in sustainable livestock production and aquaculture: a review. Ann. Anim. Sci. 19, 257-271. https://doi.org/10.2478/aoas-2018-0048 (2019).

12. Ren, H. et al. Enhanced biogas production in the duckweed anaerobic digestion process. J. Energy Resour. Technol. 140, 041805. https://doi.org/10.1115/1.4039782 (2018).

13. Cui, W. \& Cheng, J. J. Growing duckweed for biofuel production: a review. Plant Biol. 17(Suppl 1), 16-23. https://doi.org/10.1111/ plb.12216 (2015).

14. Bog, M. et al. Genetic structure of the genus Lemna L. (Lemnaceae) as revealed by amplified fragment length polymorphism. Planta 232, 609-619. https://doi.org/10.1007/s00425-010-1201-2 (2010).

15. Bog, M. et al. A taxonomic revision of Lemna sect. Uninerves (Lemnaceae). Taxon 69, 56-66. https://doi.org/10.1002/tax.12188 (2020).

16. Tippery, N. P., Les, D. H. \& Crawford, D. J. Evaluation of phylogenetic relationships in Lemnaceae using nuclear ribosomal data. Plant Biol. 17(Suppl 1), 50-58. https://doi.org/10.1111/plb.12203 (2015).

17. Bog, M. et al. Genetic characterization and barcoding of taxa in the genera Landoltia and Spirodela (Lemnaceae) by three plastidic markers and amplified fragment length polymorphism (AFLP). Hydrobiologia 749, 169-182. https://doi.org/10.1007/s10750-0142163-3 (2015).

18. Hoang, P. T. N., Schubert, V., Meister, A., Fuchs, J. \& Schubert, I. Variation in genome size, cell and nucleus volume, chromosome number and rDNA loci among duckweeds. Sci. Rep. 9, 3234. https://doi.org/10.1038/s41598-019-39332-w (2019).

19. Landolt, E. The family of Lemnaceae: a monographic study (Vol 1) (Veröffentlichungen des Geobotanischen Institutes der Eidg. Techn. Hochschule, Zürich, 1986).

20. Wang, W., Kerstetter, R. A. \& Michael, T. P. Evolution of genome size in duckweeds (Lemnaceae). J. Bot. 1-9, 2011. https://doi. org/10.1155/2011/570319 (2011).

21. Wang, W. et al. The Spirodela polyrhiza genome reveals insights into its neotenous reduction fast growth and aquatic lifestyle. Nat. Commun. 5, 3311. https://doi.org/10.1038/ncomms4311 (2014).

22. Harkess, A. et al. A new Spirodela polyrhiza genome and proteome reveal a conserved chromosomal structure with high abundances of proteins favoring energy production. J. bioRxiv https://doi.org/10.1101/2020.01.23.909457 (2020).

23. Cao, H. X. et al. The map-based genome sequence of Spirodela polyrhiza aligned with its chromosomes, a reference for karyotype evolution. New Phytol. 209, 354-363. https://doi.org/10.1111/nph.13592 (2016).

24. Hoang, P. N. T. et al. Generating a high-confidence reference genome map of the Greater Duckweed by integration of cytogenomic, optical mapping and Oxford Nanopore technologies. Plant J. 96, 670-684. https://doi.org/10.1111/tpj.14049 (2018).

25. Michael, T. P. et al. Comprehensive definition of genome features in Spirodela polyrhiza by high-depth physical mapping and short-read DNA sequencing strategies. Plant J. https://doi.org/10.1111/tpj.13400 (2017).

26. Ho, E. K. H., Bartkowska, M., Wright, S. I. \& Agrawal, A. F. Population genomics of the facultatively asexual duckweed Spirodela polyrhiza. New Phytol. 224, 1361-1371. https://doi.org/10.1111/nph.16056 (2019).

27. Xu, S. et al. Low genetic variation is associated with low mutation rate in the giant duckweed. Nat. Commun. 10, 1243. https://doi. org/10.1038/s41467-019-09235-5 (2019).

28. Chamala, S. et al. Assembly and validation of the genome of the nonmodel basal angiosperm Amborella. Science 342, 1516-1517. https://doi.org/10.1126/science.1241130 (2013). 
29. Hoang, P. T. N. \& Schubert, I. Reconstruction of chromosome rearrangements between the two most ancestral duckweed species Spirodela polyrhiza and S. intermedia. Chromosoma 126, 729-739. https://doi.org/10.1007/s00412-017-0636-7 (2017).

30. Geber, G. Zur Karyosystematik der Lemnaceae. Ph.D. thesis, University of Vienna, Vienna, Austria, p.140 (1989).

31. Urbanska-Worytkiewicz, K. Cytological variation within the family of "Lemnaceae". Veröffentlichungen des Geobotanischen Institutes der Eidg. Tech. Hochschule, Stiftung Rübel, in Zürich. https://doi.org/10.5169/seals-308615 (1980).

32. Koren, S. et al. Canu: scalable and accurate long-read assembly via adaptive k-mer weighting and repeat separation. Genome Res. 27, 722-736. https://doi.org/10.1101/gr.215087.116 (2017).

33. Darling, A. C., Mau, B., Blattner, F. R. \& Perna, N. T. Mauve: multiple alignment of conserved genomic sequence with rearrangements. Genome Res. 14, 1394-1403. https://doi.org/10.1101/gr.2289704 (2004).

34. Boetzer, M. \& Pirovano, W. SSPACE-LongRead: scaffolding bacterial draft genomes using long read sequence information. BMC Bioinform. 15, 211. https://doi.org/10.1186/1471-2105-15-211 (2014).

35. Li, H. Minimap2: pairwise alignment for nucleotide sequences. Bioinformatics 34, 3094-3100. https://doi.org/10.1093/bioinforma tics/bty191 (2018).

36. Simao, F. A., Waterhouse, R. M., Ioannidis, P., Kriventseva, E. V. \& Zdobnov, E. M. BUSCO: assessing genome assembly and annotation completeness with single-copy orthologs. Bioinformatics 31, 3210-3212. https://doi.org/10.1093/bioinformatics/btv35 1 (2015).

37. Waterhouse, R. M. et al. BUSCO applications from quality assessments to gene prediction and phylogenomics. Mol. Biol. Evol. 35, 543-548. https://doi.org/10.1093/molbev/msx319 (2018).

38. Van Hoeck, A. et al. The first draft genome of the aquatic model plant Lemna minor opens the route for future stress physiology research and biotechnological applications. Biotech. Biofuels 8, 188. https://doi.org/10.1186/s13068-015-0381-1 (2015).

39. Keilwagen, J. et al. Using intron position conservation for homology-based gene prediction. Nucl. Acids Res. 44, e89. https://doi. org/10.1093/nar/gkw092 (2016).

40. Novak, P., Neumann, P., Pech, J., Steinhaisl, J. \& Macas, J. RepeatExplorer: a Galaxy-based web server for genome-wide characterization of eukaryotic repetitive elements from next-generation sequence reads. Bioinformatics 29, 792-793. https://doi.org/10.1093/ bioinformatics/btt054 (2013).

41. Price, A. L., Jones, N. C. \& De Pevzner, P. A. novo identification of repeat families in large genomes. Bioinformatics 21(Suppl 1), i351-358. https://doi.org/10.1093/bioinformatics/bti1018 (2005).

42. Benson, G. Tandem repeats finder: a program to analyze DNA sequences. Nucl. Acids Res. 27, 573-580. https://doi.org/10.1093/ nar/27.2.573 (1999).

43. Borisjuk, N. et al. Structural and biochemical properties of duckweed surface cuticle. Front. Chem. 6, 317. https://doi.org/10.3389/ fchem.2018.00317 (2018).

44. Chakraborty, M., Baldwin-Brown, J. G., Long, A. D. \& Emerson, J. J. Contiguous and accurate de novo assembly of metazoan genomes with modest long read coverage. Nucl. Acids Res. 44, e147. https://doi.org/10.1093/nar/gkw654 (2016).

45. Jiao, W. B. et al. Improving and correcting the contiguity of long-read genome assemblies of three plant species using optical mapping and chromosome conformation capture data. Genome Res. 27, 778-786. https://doi.org/10.1101/gr.213652.116 (2017).

46. Jayakumar, V. \& Sakakibara, Y. Comprehensive evaluation of non-hybrid genome assembly tools for third-generation PacBio long-read sequence data. Br. Bioinform. 20, 866-876. https://doi.org/10.1093/bib/bbx147 (2019).

47. Maumus, F. \& Quesneville, H. Deep investigation of Arabidopsis thaliana junk DNA reveals a continuum between repetitive elements and genomic dark matter. PLoS ONE 9, e94101. https://doi.org/10.1371/journal.pone.0094101 (2014).

48. Vu, G. T. H. et al. Comparative genome analysis reveals divergent genome size evolution in a carnivorous plant genus. Plant Genome 8, 3. https://doi.org/10.3835/plantgenome2015.04.0021 (2015).

49. Schubert, I. \& Vu, G. T. H. Genome stability and evolution: attempting a holistic view. Trends Plant Sci. 21, 749-757. https://doi. org/10.1016/j.tplants.2016.06.003 (2016).

50. Gong, R. et al. Divergent functions of the GAGA-binding transcription factor family in rice. Plant J. 94, 32-47. https://doi. org/10.1111/tpj.13837 (2018).

51. Wicke, S., Costa, A., Munoz, J. \& Quandt, D. Restless 5S: the re-arrangement(s) and evolution of the nuclear ribosomal DNA in land plants. Mol. Phylogenet. Evol. 61, 321-332. https://doi.org/10.1016/j.ympev.2011.06.023 (2011).

52. Appenroth, K.-J., Teller, S. \& Horn, M. Photophysiology of turion formation and germination in Spirodela polyrhiza. Biol. Plantarum 38, 95-106. https://doi.org/10.1007/bf02879642 (1996).

53. Vondrak, T. et al. Characterization of repeat arrays in ultra-long nanopore reads reveals frequent origin of satellite DNA from retrotransposon-derived tandem repeats. Plant J. 101, 484-500. https://doi.org/10.1111/tpj.14546 (2020).

54. Zimin, A. V. et al. Hybrid assembly of the large and highly repetitive genome of Aegilops tauschii, a progenitor of bread wheat, with the MaSuRCA mega-reads algorithm. Genome Res. 27, 787-792. https://doi.org/10.1101/gr.213405.116 (2017).

55. Lagesen, K. et al. RNAmmer: consistent and rapid annotation of ribosomal RNA genes. Nucl. Acids Res. 35, 3100-3108. https:// doi.org/10.1093/nar/gkm160 (2007).

56. Chan, P. P. \& Lowe, T. M. tRNAscan-SE: Searching for tRNA genes in genomic sequences. Methods Mol. Biol. 1-14, 2019. https:// doi.org/10.1007/978-1-4939-9173-0_1 (1962).

57. Jones, P. et al. InterProScan 5: genome-scale protein function classification. Bioinformatics 30, 1236-1240. https://doi.org/10.1093/ bioinformatics/btu031 (2014).

58. Huerta-Cepas, J. et al. Fast genome-wide functional annotation through orthology assignment by eggNOG-Mapper. Mol. Biol. Evol. 34, 2115-2122. https://doi.org/10.1093/molbev/msx148 (2017).

59. Huerta-Cepas, J. et al. eggNOG 4.5: a hierarchical orthology framework with improved functional annotations for eukaryotic, prokaryotic and viral sequences. Nucl. Acids Res. 44, 286-293. https://doi.org/10.1093/nar/gkv1248 (2016).

60. Neph, S. et al. BEDOPS: high-performance genomic feature operations. Bioinformatics 28, 1919-1920. https://doi.org/10.1093/ bioinformatics/bts277 (2012).

61. Quinlan, A. R. \& Hall, I. M. BEDTools: a flexible suite of utilities for comparing genomic features. Bioinformatics 26, 841-842. https://doi.org/10.1093/bioinformatics/btq033 (2010).

62. Krzywinski, M. et al. Circos: an information aesthetic for comparative genomics. Genome Res. 19, 1639-1645. https://doi. org/10.1101/gr.092759.109 (2009).

63. Neumann, P., Novak, P., Hostakova, N. \& Macas, J. Systematic survey of plant LTR-retrotransposons elucidates phylogenetic relationships of their polyprotein domains and provides a reference for element classification. Mob. DNA 10, 1. https://doi.org/10.1186/ s13100-018-0144-1 (2019).

64. Sobreira, T. J., Durham, A. M. \& Gruber, A. TRAP: automated classification, quantification and annotation of tandemly repeated sequences. Bioinformatics 22, 361-362. https://doi.org/10.1093/bioinformatics/bti809 (2006).

65. Kuzoff, R. K., Sweere, J. A., Soltis, D. E., Soltis, P. S. \& Zimmer, E. A. The phylogenetic potential of entire 26S rDNA sequences in plants. Mol. Biol. Evol. 15, 251-263 (1998).

66. Shoup, S. \& Lewis, L. A. Polyphyletic origin of parallel basal bodies in swimming cells of chlorophycean green algae (Chlorophyta). J. Phycol. 39, 789-796. https://doi.org/10.1046/j.1529-8817.2003.03009.x (2003).

67. Lysak, M. A. et al. Mechanisms of chromosome number reduction in Arabidopsis thaliana and related Brassicaceae species. Proc. Nat. Acad. Sci. USA 103, 5224-5229. https://doi.org/10.1073/pnas.0510791103 (2006). 


\title{
Acknowledgements
}

We thank Todd Michael (JCVI, San Diego, USA) for providing repeat data for S. polyrhiza, Andrea Koblížková for assistance in DNA isolation for Oxford Nanopore sequencing and Klaus-J. Appenroth University of Jena, for critical remarks. This work was supported by grants of the German Research Foundation [SCHU 951/181] to IS, and of the Czech Academy of Sciences [RVO:60077344] to JM and PN. PNTH was supported by the Vietnam National Foundation for Science and Technology Development (NAFOSTED) Grant \# 106.01-2020.33. Computing and data storage facilities were in part supported by the ELIXIR-CZ research infrastructure Project [MEYS No: LM2015047].

\section{Author contributions}

P.N.T.H., A.F., P.N., J.M., I.S., N.B. designed experiments; P.N.T.H. performed cytogenetic experiments, H.X.C. isolated DNA for PacBio sequencing, A.S., G.C., N.B. performed molecular analyses of rDNA, J.M. performed Oxford Nanopore sequencing, A.F., P.N. preformed genome assemblies, P.N.T.H., A.F., U.S., J.M., P.N., I.S. analyzed data; P.N.T.H., A.F., N.B., J.M., I.S. wrote the manuscript. All authors read and approved the manuscript.

\section{Funding}

Open Access funding enabled and organized by Projekt DEAL.

\section{Competing interests}

The authors declare no competing interests.

\section{Additional information}

Supplementary information is available for this paper at https://doi.org/10.1038/s41598-020-75728-9.

Correspondence and requests for materials should be addressed to I.S.

Reprints and permissions information is available at www.nature.com/reprints.

Publisher's note Springer Nature remains neutral with regard to jurisdictional claims in published maps and institutional affiliations.

\begin{abstract}
(c) (i) Open Access This article is licensed under a Creative Commons Attribution 4.0 International License, which permits use, sharing, adaptation, distribution and reproduction in any medium or format, as long as you give appropriate credit to the original author(s) and the source, provide a link to the Creative Commons licence, and indicate if changes were made. The images or other third party material in this article are included in the article's Creative Commons licence, unless indicated otherwise in a credit line to the material. If material is not included in the article's Creative Commons licence and your intended use is not permitted by statutory regulation or exceeds the permitted use, you will need to obtain permission directly from the copyright holder. To view a copy of this licence, visit http://creativecommons.org/licenses/by/4.0/.
\end{abstract}

(c) The Author(s) 2020 